\title{
Periodic nonlinear sliding modes for two uniformly magnetized spheres
}

Boyd F. Edwards, and John M. Edwards

Citation: Chaos 27, 053107 (2017); doi: 10.1063/1.4982202

View online: https://doi.org/10.1063/1.4982202

View Table of Contents: http://aip.scitation.org/toc/cha/27/5

Published by the American Institute of Physics

\section{Articles you may be interested in}

Experimental analysis of density fingering instability modified by precipitation

Chaos: An Interdisciplinary Journal of Nonlinear Science 27, 053111 (2017); 10.1063/1.4983670

Synchronization of moving oscillators in three dimensional space

Chaos: An Interdisciplinary Journal of Nonlinear Science 27, 053115 (2017); 10.1063/1.4984026

Calculation of Hamilton energy and control of dynamical systems with different types of attractors

Chaos: An Interdisciplinary Journal of Nonlinear Science 27, 053108 (2017); 10.1063/1.4983469

The stability of fixed points for a Kuramoto model with Hebbian interactions

Chaos: An Interdisciplinary Journal of Nonlinear Science 27, 053110 (2017); 10.1063/1.4983524

On the influence of additive and multiplicative noise on holes in dissipative systems

Chaos: An Interdisciplinary Journal of Nonlinear Science 27, 053101 (2017); 10.1063/1.4982205

A critical comparison of Lagrangian methods for coherent structure detection

Chaos: An Interdisciplinary Journal of Nonlinear Science 27, 053104 (2017); 10.1063/1.4982720 


\title{
Periodic nonlinear sliding modes for two uniformly magnetized spheres
}

\author{
Boyd F. Edwards ${ }^{1}$ and John M. Edwards ${ }^{2}$ \\ ${ }^{1}$ Department of Physics, Utah State University, Logan, Utah 84322, USA \\ ${ }^{2}$ Department of Informatics and Computer Science, Idaho State University, Pocatello, Idaho 83209, USA
}

(Received 8 December 2016; accepted 12 April 2017; published online 12 May 2017)

\begin{abstract}
A uniformly magnetized sphere slides without friction along the surface of a second, identical sphere that is held fixed in space, subject to the magnetic force and torque of the fixed sphere and the normal force. The free sphere has two stable equilibrium positions and two unstable equilibrium positions. Two small-amplitude oscillatory modes describe the sliding motion of the free sphere near each stable equilibrium, and an unstable oscillatory mode describes the motion near each unstable equilibrium. The three oscillatory modes remain periodic at finite amplitudes, one bifurcating into mixed modes and circumnavigating the free sphere at large energies. For small energies, the free sphere is confined to one of the two discontiguous domains, each surrounding a stable equilibrium position. At large energies, these domains merge and the free sphere may visit both positions. The critical energy at which these domains merge coincides with the cumulation point of an infinite cascade of mixed-mode bifurcations. These findings exploit the equivalence of the force and torque between two uniformly magnetized spheres and the force and torque between two equivalent point dipoles, and offer clues to the rich nonlinear dynamics of this system. Online MagPhyx visualizations illustrate the dynamics. Published by AIP Publishing. [http://dx.doi.org/10.1063/1.4982202]
\end{abstract}

Magnetic interactions have been studied systematically for centuries. Yet calculating magnetic forces and torques, which are simple for two point dipoles, generally requires numerical or approximate analytical techniques for extended objects. We showed recently that magnetic interactions between two uniformly magnetized spheres are identical to the interactions between two point magnetic dipoles. This equivalence opens the door to simple, exact studies of nonlinear dynamical interactions between extended magnets. In this paper, we explore the motion of a uniformly magnetized sphere in response to the magnetic forces and torques produced by a second, fixed sphere. We derive the Hamiltonian and consider periodic orbits for which the free sphere maintains contact with the fixed sphere, sliding along its surface without friction. For low energies, the free sphere is confined to the vicinity of one of its two stable equilibrium positions. For higher energies, the free sphere may visit both positions. The critical energy marks the cumulation point of an infinite cascade of periodic finite-amplitude modes. The work was originally motivated by interest in the interactions between collections of small neodymium magnet spheres used as desk toys.

\section{INTRODUCTION}

Collections of small neodymium magnet spheres are used as a creative medium to build beautiful sculptures, some made from thousands of magnets, including models of molecules, fractals, and Platonic solids. ${ }^{1}$ These spheres have spawned a learning community dedicated to sharing photos and tutorials of magnetic sculptures, with YouTube tutorial videos attracting over a hundred million views. ${ }^{2}$ These spheres offer engaging hands-on exposure to principles of magnetism, and are used both in and out of the classroom to teach principles of mathematics, physics, chemistry, biology, and engineering. ${ }^{2,3}$

This general interest has motivated scientific studies of the behavior of assemblies of spherical magnets. These studies address the dynamics of chain, ring, and tube formation, ${ }^{4-6}$ a continuum model for magnet chain energy, ${ }^{7}$ mechanical properties of chains and cylinders, ${ }^{8}$ stable defects along chains and rings, ${ }^{9}$ eigenmodes for lateral oscillations of a straight chain, ${ }^{10}$ and stability of vertical chains. ${ }^{11}$ These studies emphasize the energetics and stability of symmetric assemblies of many spherical magnets. Yet even small numbers of magnets can exhibit complex non-symmetric translations and rotations as they seek a stable equilibrium state.

In this paper, we consider the simplest possible case of two magnets remaining in frictionless contact at all times, with one magnet fixed and the other free to slide along the surface of the other, with their magnetic moments in the same plane, and with the position and magnetic orientation of the free sphere specified by angles $\theta$ and $\phi$, respectively. The corresponding orbital and spin momenta $p_{\theta}$ and $p_{\phi}$ bring the phase-space dimensionality to four, and energy conservation supplies an algebraic relationship between these four dynamical variables that reduces the effective dimensionality to three.

This sliding two-magnet system has the same phasespace dimensionality as the double-pendulum problem, ${ }^{12-14}$ and offers simpler algebra, closed-form results for normal modes and domain boundaries, finite-amplitude results for normal modes, and an infinite cascade of mixed-mode bifurcations culminating in a transition from oscillatory motion to orbital motion. The two-magnet system also offers a straightforward extension to a six-dimensional problem with radial motion and sphere collisions, in addition to orbital and spin motions. 
A solid sphere of radius $a$ and uniform magnetization $\mathbf{M}$ has total magnetic dipole moment

$$
\mathbf{m}_{1}=\frac{4}{3} \pi a^{3} \mathbf{M}
$$

At a point $\mathbf{r}$ outside of a uniformly magnetized sphere centered at the origin, its magnetic field is a pure dipolar field given by ${ }^{15-18}$

$$
\mathbf{B}(\mathbf{r})=\frac{\mu_{0}}{4 \pi}\left(\frac{3 \mathbf{m}_{1} \cdot \mathbf{r}}{r^{5}} \mathbf{r}-\frac{\mathbf{m}_{1}}{r^{3}}\right),
$$

where $r=|\mathbf{r}|>a$.

We showed recently that this field exerts forces and torques on a second uniformly magnetized sphere that are identical to the forces and torques on a point dipole. ${ }^{19,20}$ Namely, for a second sphere of total dipole moment $\mathbf{m}_{2}$ centered at position $\mathbf{r}$, the energy of interaction, force, and torque are, respectively,

$$
\begin{gathered}
U=-\mathbf{m}_{2} \cdot \mathbf{B}, \\
\mathbf{F}=-\nabla U, \\
\tau=\mathbf{m}_{2} \times \mathbf{B},
\end{gathered}
$$

where $\mathbf{B}$ is given by Eq. (2).

In this paper, we exploit this point-sphere equivalence to study the 2D dynamics of two identical, uniformly magnetized spheres of radius $a$, dipole moment magnitude $m$, mass $\tilde{m}$, and moment of inertia $I=\frac{2}{5} \tilde{m} a^{2}$. Sphere 1 is fixed at the origin, with magnetic moment $\mathbf{m}_{1}=m \hat{\mathbf{x}}$. Sphere 2 is free to move about a plane in response to the forces and torques produced by the fixed sphere. We use polar coordinates $(r, \theta)$ to describe the position $\mathbf{r}=r \cos \theta \hat{\mathbf{x}}+r \sin \theta \hat{\mathbf{y}}$ of the center of the free sphere, and angle $\phi$ to describe the angle of orientation of its dipole moment, given by $\mathbf{m}_{2}=m \cos \phi \hat{\mathbf{x}}+$ $m \sin \phi \hat{\mathbf{y}}$ (Fig. 1).

For this geometry, Eqs. (2)-(5) become

$$
\begin{gathered}
\mathbf{B}=\frac{\mu_{0} m}{8 \pi r^{3}}[(1+3 \cos 2 \theta) \hat{\mathbf{x}}+3 \sin 2 \theta \hat{\mathbf{y}}], \\
U=-\frac{\mu_{0} m^{2}}{8 \pi r^{3}}[\cos \phi+3 \cos (\phi-2 \theta)] \\
\mathbf{F}=-\frac{\partial U}{\partial r} \hat{\mathbf{r}}-\frac{1}{r} \frac{\partial U}{\partial \theta} \hat{\boldsymbol{\theta}} \\
\tau=-\frac{\mu_{0} m^{2}}{8 \pi r^{3}}[\sin \phi+3 \sin (\phi-2 \theta)] \hat{\mathbf{z}}
\end{gathered}
$$

where

$$
\begin{gathered}
\hat{\mathbf{r}}=\cos \theta \hat{\mathbf{x}}+\sin \theta \hat{\mathbf{y}}, \\
\hat{\boldsymbol{\theta}}=-\sin \theta \hat{\mathbf{x}}+\cos \theta \hat{\mathbf{y}}
\end{gathered}
$$

are the polar unit vectors.

We approach the problem by writing its Hamiltonian (Sec. II), defining dimensionless variables (Sec. III), finding the equilibrium positions of the free sphere (Sec. IV), determining the spatial domain(s) to which the free sphere is

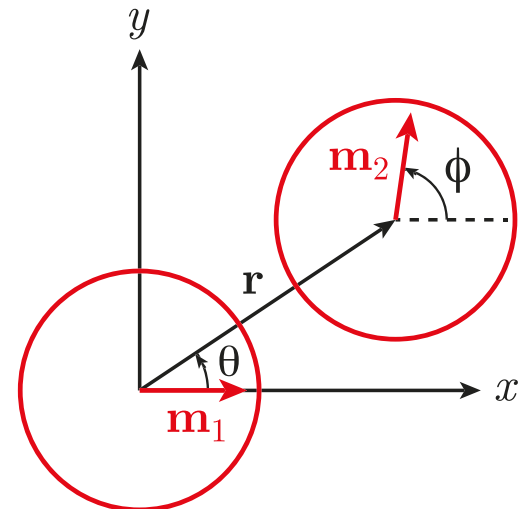

FIG. 1. Polar coordinate system $(r, \theta)$ used to describe the 2D position of a free sphere with magnetic dipole moment $\mathbf{m}_{2}$, whose direction is given by the angle $\phi$. This sphere moves in response to the forces and torques produced by a sphere that is held fixed at the origin, with its magnetic moment $\mathbf{m}_{1}$ in the $+x$ direction. Reproduced with permission from B. F. Edwards and J. M. Edwards, Eur. J. Phys. 38, 015205 (2017). Copyright 2017 IOP Publishing.

confined (Sec. V), specializing to sliding solutions for which the two spheres are always in contact with each other, and involving only orbital and spin motions (Sec. VI), analyzing the linear stability of the equilibrium positions for such sliding solutions (Sec. VII), exploring finite-amplitude periodic sliding solutions (Sec. VIII), and concluding with suggestions for further explorations (Sec. IX).

\section{HAMILTONIAN}

The Hamiltonian formalism supplies an elegant description of the dynamics. The total kinetic energy of the free sphere

$$
T=\frac{\tilde{m}}{2} \dot{r}^{2}+\frac{\tilde{m}}{2} r^{2} \dot{\theta}^{2}+\frac{I}{2} \dot{\phi}^{2}
$$

includes, from left to right, the radial kinetic energy, the orbital kinetic energy, and the spin kinetic energy. The Lagrangian of the free sphere

$$
L=T-U
$$

is a function of the coordinates $r, \theta$, and $\phi$ and the velocities $\dot{r}=d r / d t, \dot{\theta}=d \theta / d t$, and $\dot{\phi}=d \phi / d t$. The momenta

$$
\begin{gathered}
p_{r}=\frac{\partial L}{\partial \dot{r}}=\tilde{m} \dot{r} \\
p_{\theta}=\frac{\partial L}{\partial \dot{\theta}}=\tilde{m} r^{2} \dot{\theta}, \\
p_{\phi}=\frac{\partial L}{\partial \dot{\phi}}=I \dot{\phi}
\end{gathered}
$$

give the respective radial momentum, orbital angular momentum, and spin angular momentum of the free sphere.

The Legendre transformation $H=\dot{r} p_{r}+\dot{\theta} p_{\theta}+\dot{\phi} p_{\phi}-L$ yields the Hamiltonian

$$
H=\frac{p_{r}^{2}}{2 \tilde{m}}+\frac{p_{\theta}^{2}}{2 \tilde{m} r^{2}}+\frac{p_{\phi}^{2}}{2 I}+U(r, \theta, \phi),
$$


a function of the coordinates and the momenta. Because the coordinate transformations have no explicit time dependence, $H=T+U$ is the total energy $E$. Because $H$ has no explicit time dependence, it is a constant of the motion. Because $L$ depends explicitly on $r, \theta$, and $\phi$, none of the momenta are constants of the motion, whence momentum and energy may be exchanged between radial, orbital, and spin motions.

Partial derivatives of the Hamiltonian give the equations of motion

$$
\begin{gathered}
\dot{r}=\frac{\partial H}{\partial p_{r}}=\frac{p_{r}}{\tilde{m}}, \\
\dot{\theta}=\frac{\partial H}{\partial p_{\theta}}=\frac{p_{\theta}}{\tilde{m} r^{2}}, \\
\dot{\phi}=\frac{\partial H}{\partial p_{\phi}}=\frac{p_{\phi}}{I}, \\
\dot{p}_{r}=-\frac{\partial H}{\partial r}=\frac{p_{\theta}^{2}}{\tilde{m} r^{3}}-\frac{\partial U}{\partial r}, \\
\dot{p}_{\theta}=-\frac{\partial H}{\partial \theta}=-\frac{\partial U}{\partial \theta}, \\
\dot{p}_{\phi}=-\frac{\partial H}{\partial \phi}=-\frac{\partial U}{\partial \phi},
\end{gathered}
$$

with $U=U(r, \theta, \phi)$ given by Eq. (7).

Equations (18)-(23) are equivalent to a Newtonian formulation with

$$
\begin{aligned}
\tilde{m} \dot{\mathbf{v}} & =\mathbf{F}, \\
I \dot{\omega} & =\tau,
\end{aligned}
$$

where $\mathbf{v}=\dot{\mathbf{r}}$ and $\omega=\dot{\phi} \hat{\mathbf{z}}$ are the linear and angular velocities. Inserting Eq. (8) into Eq. (24) gives Eqs. (21) and (22), the radial and angular components of the force, with the first term on the right side of Eq. (21) giving the centrifugal force. Inserting Eq. (9) into Eq. (25) gives Eq. (23), the torque.

The Hamiltonian approach accounts for both the force and the torque through the potential energy $U$ given by Eq. (7). This is no surprise for the force, which satisfies $\mathbf{F}=-\nabla U$. For the torque, though, the expression $\tau=\mathbf{m}_{2} \times \mathbf{B}$ belies its relationship with the potential energy. This expression leads to Eq. (9), which must be invoked explicitly in the Newtonian approach, but is not needed in the Hamiltonian approach. Comparison with Eq. (7) allows Eq. (9) to be rewritten as

$$
\tau=-\frac{\partial U}{\partial \phi} \hat{\mathbf{z}}
$$

which suggests a relationship between the torque and the potential energy. Equation (23) of the Hamiltonian formulation confirms this relationship. ${ }^{21}$

When the free sphere comes in contact with the fixed sphere, the free sphere experiences a normal force $\mathbf{F}_{\mathrm{N}}=F_{\mathrm{N}} \hat{\mathbf{r}}$ in the radial direction, with $F_{\mathrm{N}}>0$ to prevent the two hard spheres from overlapping. Including this force in Eqs. (24) and (21) gives

$$
\begin{gathered}
\tilde{m} \dot{\mathbf{v}}=\mathbf{F}+\mathbf{F}_{\mathrm{N}}, \\
\dot{p}_{r}=-\frac{\partial H}{\partial r}=\frac{p_{\theta}^{2}}{\tilde{m} r^{3}}-\frac{\partial U}{\partial r}+F_{\mathrm{N}} .
\end{gathered}
$$

\section{DIMENSIONLESS VARIABLES}

To simplify calculations, we scale length by the magnet diameter $D=2 a$, force by $F_{0}=3 \mu_{0} \mathrm{~m}^{2} / 2 \pi D^{4}$, energy and torque by $F_{0} D$, time by $T_{0}=\sqrt{\tilde{m} D / F_{0}}$, magnetic field by $F_{0} D / m$, magnetic moment by $m$, velocity by $D / T_{0}$, acceleration by $D / T_{0}^{2}$, angular velocity by $T_{0}^{-1}$, angular acceleration by $T_{0}^{-2}$, radial (linear) momentum by $\tilde{m} D / T_{0}$, and orbital and spin angular momentum by $\tilde{m} D^{2} / T_{0}$. In dimensionless units, the magnet diameter and the center-to-center distance between touching magnets are both 1 , and the magnetic moment vectors are unit vectors.

In dimensionless variables, the Hamiltonian and dynamical equations become

$$
\begin{gathered}
E=T+U, \\
T=\frac{p_{r}^{2}}{2}+\frac{p_{\theta}^{2}}{2 r^{2}}+5 p_{\phi}^{2}, \\
U=-\frac{1}{12 r^{3}}[\cos \phi+3 \cos (\phi-2 \theta)], \\
\dot{r}=p_{r}, \\
\dot{\theta}=\frac{p_{\theta}}{r^{2}} \\
\dot{\phi}=10 p_{\phi}, \\
\dot{p}_{r}=\frac{p_{\theta}^{2}}{r^{3}}+\frac{3 U}{r}+F_{\mathrm{N}}, \\
\dot{p}_{\theta}=-\frac{\partial U}{\partial \theta}=\frac{1}{2 r^{3}} \sin (\phi-2 \theta), \\
\frac{\partial U}{\partial \phi}=-\frac{1}{12 r^{3}}[\sin \phi+3 \sin (\phi-2 \theta)],
\end{gathered}
$$

where we have included the normal force of Eq. (28).

The dimensionless magnetic force and torque are given by

$$
\begin{gathered}
\mathbf{F}=-\frac{\partial U}{\partial r} \hat{\mathbf{r}}-\frac{1}{r} \frac{\partial U}{\partial \theta} \hat{\theta}, \\
\tau=-\frac{\partial U}{\partial \phi} \hat{\mathbf{z}} .
\end{gathered}
$$

The dimensionless magnetic field of the fixed sphere

$$
\mathbf{B}=\frac{1}{12 r^{3}}[(1+3 \cos 2 \theta) \hat{\mathbf{x}}+3 \sin 2 \theta \hat{\mathbf{y}}]
$$

has the magnitude

$$
B(r, \theta)=\frac{1}{12 r^{3}}(10+6 \cos 2 \theta)^{1 / 2}
$$

and the direction 


$$
\phi_{m}(\theta)=\tan ^{-1}\left(\frac{3 \sin 2 \theta}{1+3 \cos 2 \theta}\right)
$$

The dimensionless magnetic moment of the free sphere

$$
\mathbf{m}_{2}=\cos \phi \hat{\mathbf{x}}+\sin \phi \hat{\mathbf{y}}
$$

has the magnitude $m_{2}=1$ and the direction $\phi$.

Defining the angle $\beta$ between $\mathbf{m}_{2}$ and $\mathbf{B}$ as

$$
\beta=\phi-\phi_{m},
$$

we can write the dimensionless potential energy as

$$
U(r, \theta, \phi)=-\mathbf{m}_{2} \cdot \mathbf{B}=-B \cos \beta,
$$

which replicates Eq. (31). At a particular position $(r, \theta)$, the minimum potential energy

$$
U_{\mathrm{m}}(r, \theta)=U\left(r, \theta, \phi_{m}\right)=-B(r, \theta)
$$

follows by setting $\phi=\phi_{m}$ in Eq. (31) or by setting $\beta=0$ in Eq. (45), where $B(r, \theta)$ is given by Eq. (41). Thus, orienting $\mathbf{m}_{2}$ in the direction of $\mathbf{B}$ minimizes the potential energy.

We use dimensionless variables hereafter.

\section{EQUILIBRIUM POSITIONS}

Equations (29)-(37) admit four equilibrium positions $(r, \theta, \phi)$ of the free sphere.

At positions $\mathrm{X}^{+}=(1,0,0)$ and $\mathrm{X}^{-}=(1, \pi, 0)$, the dipole moments $\mathbf{m}_{1}$ and $\mathbf{m}_{2}$ of the two magnets are parallel, and the north pole of one magnet contacts, and attracts, the south pole of the other (Fig. 2). The potential energy $U=-1 / 3$ at these positions minimizes $U_{m}(1, \theta)$ of Eq. (46) with respect to $\theta$. As shown below, these equilibrium positions are stable.

At positions $\mathrm{Y}^{+}=(1, \pi / 2, \pi)$ and $\mathrm{Y}^{-}=(1,-\pi / 2, \pi)$, the dipole moments are antiparallel, the magnets contact each

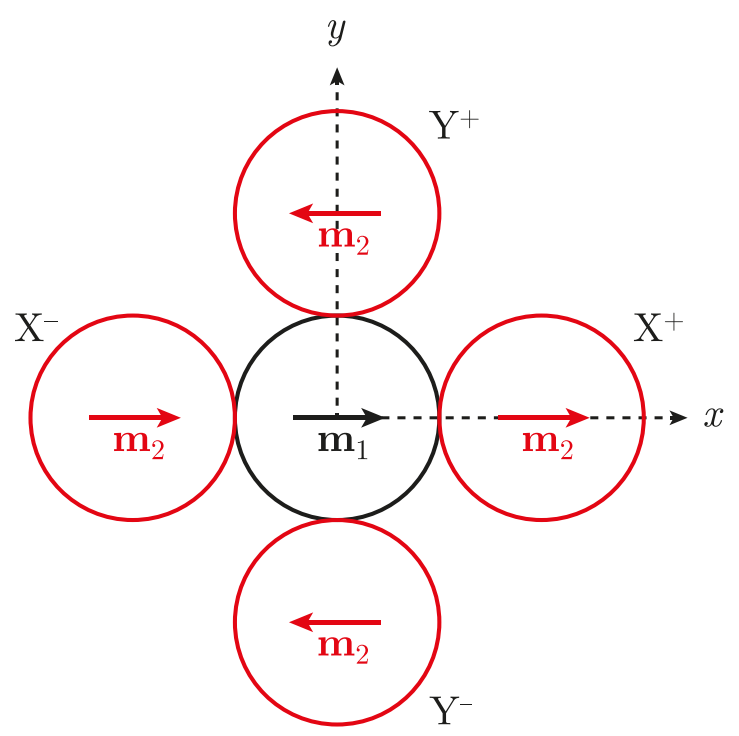

FIG. 2. Four equilibrium positions of a free sphere with magnetic dipole moment $\mathbf{m}_{2}$ surrounding a fixed sphere with moment $\mathbf{m}_{1}$ centered at the origin. At positions $\mathrm{X}^{ \pm}, \mathbf{m}_{1}$ and $\mathbf{m}_{2}$ are parallel, and at positions $\mathrm{Y}^{ \pm}$, these moments are antiparallel. other at points on their magnetic equators, and the north and south poles of each magnet contribute to the attraction between them (Fig. 2). The potential energy $U=-1 / 6$ at these positions maximizes $U_{m}(1, \theta)$ with respect to $\theta$. As shown below, these equilibrium positions are unstable.

For $E>-1 / 3$, the free sphere cannot remain stationary at $\mathrm{X}^{ \pm}$, but can pass through this point. And for $E>-1 / 6$, the free sphere cannot remain stationary at $\mathrm{Y}^{ \pm}$, but can pass through this point. For the free sphere to maintain contact with the fixed sphere when the free sphere passes through these positions, Eq. (35) and $F_{\mathrm{N}}>0$ require

$$
U<-\frac{p_{\theta}^{2}}{3} .
$$

\section{DOMAIN BOUNDARIES}

The total energy $E=T+U$ determines the domain of the free sphere, that is, the regions of space to which it is confined. Because $T \geq 0$, a location $(r, \theta)$ is accessible only if $E \geq U_{\mathrm{m}}(r, \theta)$. Setting $E=U_{\mathrm{m}}\left(r_{c}, \theta\right)$ and applying Eqs. (41) and (46) gives the maximum radius

$$
r_{c}(\theta)=\frac{(10+6 \cos 2 \theta)^{1 / 6}}{(-12 E)^{1 / 3}}
$$

The minimum radius is $r=1$, where the free sphere contacts the fixed sphere. Thus, the free sphere is confined to the domain $1 \leq r \leq r_{c}(\theta)$.

At the minimum energy $E=-1 / 3$, the free sphere remains stationary at $\mathrm{X}^{+}$or $\mathrm{X}^{-}$because $r_{c}(0)=r_{c}(\pi)=1$. Thus, $\mathrm{X}^{ \pm}$are fixed points for $E=-1 / 3$.

For $-1 / 3<E<-1 / 6$, sphere 2 is confined to the vicinity of either $\mathrm{X}^{+}$or $\mathrm{X}^{-}$and cannot overcome the potential barriers at $\mathrm{Y}^{ \pm}$. For $\mathrm{X}^{+}, \theta$ is confined to the range $|\theta|<\theta_{c}$, and for $\mathrm{X}^{-}$, the range $|\theta-\pi|<\theta_{c}$, where $\theta_{c}$ is an acute angle given by setting $r_{c}\left(\theta_{c}\right)=1$ in Eq. (48), yielding

$$
\cos 2 \theta_{c}=24 E^{2}-\frac{5}{3} .
$$

The value of $\theta_{c}$ increases from 0 to $\pi / 2$ as $E$ increases from $-1 / 3$ to $-1 / 6$.

$E=-1 / 6$ marks the threshold between discontiguous domains for $E<-1 / 6$ and contiguous domains for $E>-1 / 6$.

For $-1 / 6<E<0, r_{c}(\theta)>1$ is defined for all $\theta$. The free sphere has sufficient energy to surmount the potential barriers at $\mathrm{Y}^{ \pm}$and to visit both $\mathrm{X}^{+}$and $\mathrm{X}^{-}$. The domain is contiguous and finite.

For $E \geq 0, r_{c}(\theta)$ is undefined for all $\theta$ and the domain is contiguous and infinite. The free sphere is unbounded.

Figure 3 shows domain boundaries for $E=n / 12$, including the stable equilibrium positions $\mathrm{X}^{ \pm}$for $n=-4$, discontiguous domains for $n=-3$, the threshold domain for $n=-2$, and a contiguous domain for $n=-1$.

Of interest are the bounding values of $\beta=\phi-\phi_{m}$ when the spheres are in contact with each other $(r=1)$. Setting $U\left(1, \theta, \phi_{m}+\beta_{c}\right)=E$ in Eq. (31) and applying Eq. (42) gives 


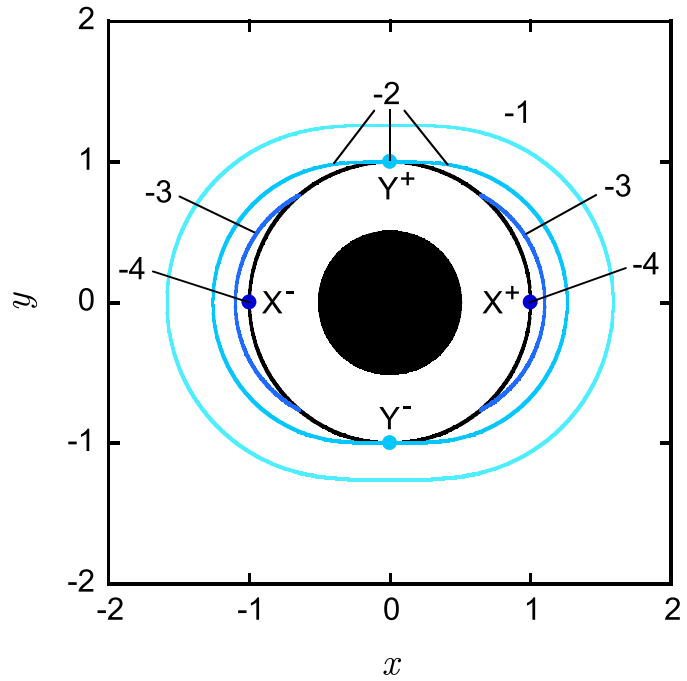

FIG. 3. Dimensionless outer domain boundaries $r_{c}(\theta)$ for the center of the free sphere for bound orbits with energies $E=n / 12$, for $n=-1,-2$, and -3 [numbered traces, from Eq. (48)]. When $n=-4$, the energy reaches its minimum and the domain shrinks to two points, the stable equilibrium positions $\mathrm{X}^{ \pm}$, shown as dots. Also shown as dots are the unstable equilibrium positions $\mathrm{Y}^{ \pm}$, with $n=-2$. The fixed sphere, of diameter 1 , is shown as a disk centered at the origin, surrounded by a circle of radius 1 that serves as the inner domain boundary for the center of the free sphere, also of diameter 1.

$$
\cos \beta_{c}=r_{c}^{-3}(\theta)
$$

Values of $\beta$ satisfy $|\beta|<\beta_{c}$, as illustrated in Fig. 4, which shows domain boundaries for $E=n / 12$, including the equilibrium points $\mathrm{X}^{ \pm}$for $n=-4$, discontiguous domains for $n=-3$, the threshold domain for $n=-2$, and contiguous domains for $n=-1,0,1,2$, and 3 . Values of $\phi$ satisfy $\left|\phi-\phi_{m}\right|<\beta_{c}$, implying that the lower the energy $E$, the closer the alignment of the free-sphere magnetic moment to the field.

\section{SLIDING SOLUTIONS}

We now specialize to solutions for which the free sphere begins and remains in contact with the fixed sphere, resulting in sliding motions of the free sphere against the fixed sphere. The values $r=1$ and $p_{r}=0$ imply that the free sphere contacts the fixed sphere and has no radial momentum. If these conditions are satisfied initially, the spheres will remain in contact as long as Eq. (47) continues to hold. We accordingly ignore Eqs. (32) and (35), and rewrite Eqs. (29)-(37) as

$$
\begin{gathered}
E=\frac{p_{\theta}^{2}}{2}+5 p_{\phi}^{2}+U \\
U=-\frac{1}{12}[\cos \phi+3 \cos (\phi-2 \theta)], \\
\dot{\theta}=p_{\theta}, \\
\dot{\phi}=10 p_{\phi}, \\
\dot{p}_{\theta}=\frac{1}{2} \sin (\phi-2 \theta), \\
\dot{p}_{\phi}=-\frac{1}{12}[\sin \phi+3 \sin (\phi-2 \theta)] .
\end{gathered}
$$

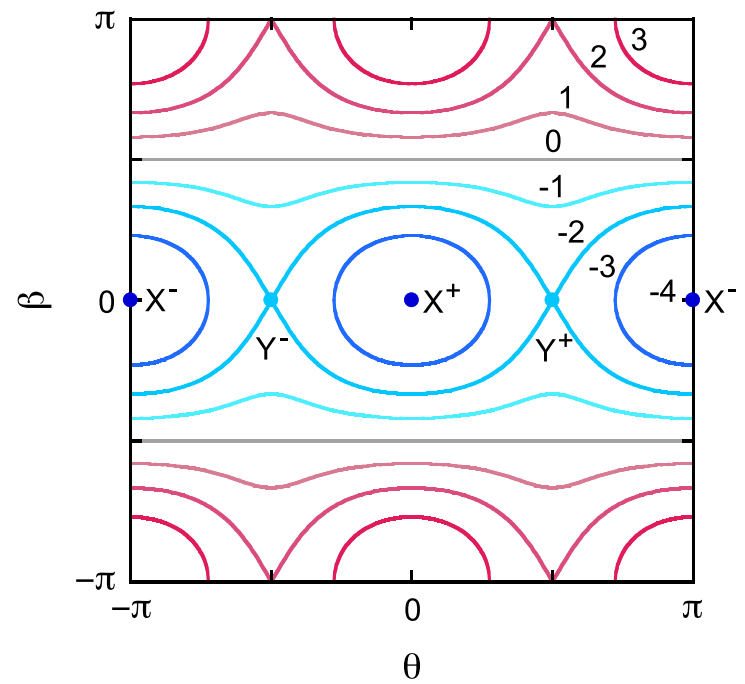

FIG. 4. Domain boundaries $\beta= \pm \beta_{c}$ versus $\theta$ for $r=1$ and energies $E=n /$ 12 , with $n=-3,-2, \ldots, 3$ [numbered traces, from Eq. (50)]. Shown as dots are the equilibrium positions $\mathrm{X}^{ \pm}(n=-4)$ and $\mathrm{Y}^{ \pm}(n=-2)$.

A four-dimensional phase space describes the timedependent solutions, which take the vector form

$$
\mathbf{x}=\left(\begin{array}{c}
\theta \\
\phi \\
p_{\theta} \\
p_{\phi}
\end{array}\right)
$$

These solutions must satisfy the energy constraint $E=E(\mathbf{x})$ $=$ constant, which effectively reduces the phase space dimensionality to three.

Equations (51)-(56) describe a system that is mathematically simpler than the double pendulum problem, which has the same phase-space dimensionality. ${ }^{12-14}$ As will be seen below, this simplicity enables closed-form solutions for domain boundaries, fixed points, and small-amplitude mode frequencies, eigenvectors, amplitudes, and stable and unstable manifolds.

\section{FIXED POINTS}

Fixed points are values of the dynamical variables for which $\dot{\mathbf{x}}=0$, and are denoted by

$$
\mathbf{x}^{*}=\left(\begin{array}{c}
\theta^{*} \\
\phi^{*} \\
p_{\theta}^{*} \\
p_{\phi}^{*}
\end{array}\right) .
$$

For these fixed points, Eqs. (51)-(56) require

$$
\begin{aligned}
E^{*} & =U, \\
U^{*}=-\frac{1}{12}\left[\cos \phi^{*}\right. & \left.+3 \cos \left(\phi^{*}-2 \theta^{*}\right)\right], \\
p_{\theta}^{*} & =0, \\
p_{\phi}^{*} & =0,
\end{aligned}
$$




$$
\begin{gathered}
\sin \left(\phi^{*}-2 \theta^{*}\right)=0, \\
\sin \phi^{*}=0,
\end{gathered}
$$

and Eq. (47) requires $U^{*}<0$.

Four fixed points satisfy these conditions. Two fixed points

$$
\mathbf{x}_{\mathrm{X}^{ \pm}}^{*}=\left(\begin{array}{c}
\pi / 2 \mp \pi / 2 \\
0 \\
0 \\
0
\end{array}\right)
$$

are located at the equilibrium positions $\mathrm{X}^{ \pm}$and have energies $E^{*}=U^{*}=-1 / 3$. Two other fixed points

$$
\mathbf{x}_{\mathrm{Y}^{ \pm}}^{*}=\left(\begin{array}{c} 
\pm \pi / 2 \\
\pi \\
0 \\
0
\end{array}\right)
$$

are located at the equilibrium positions $\mathrm{Y}^{ \pm}$and have energies $E^{*}=U^{*}=-1 / 6$.

\section{A. Fixed point stability}

We investigate the behavior near these fixed points, and their stability, by considering small-amplitude perturbations of the form

$$
\mathbf{x}^{\prime}(t)=\left(\begin{array}{c}
\theta^{\prime} \\
\phi^{\prime} \\
p_{\theta}^{\prime} \\
p_{\phi}^{\prime}
\end{array}\right)
$$

We substitute

$$
\mathbf{x}(t)=\mathbf{x}^{*}+\mathbf{x}^{\prime}(t)
$$

into Eqs. (53)-(56) and linearize in the primed perturbations, yielding

$$
\dot{\mathbf{x}}^{\prime}=A \mathbf{x}^{\prime}
$$

and

$$
A=\left(\begin{array}{cccc}
0 & 0 & 1 & 0 \\
0 & 0 & 0 & 10 \\
-1 & 1 / 2 & 0 & 0 \\
1 / 2 & E^{*} & 0 & 0
\end{array}\right)
$$

where $E^{*}=-1 / 3$ for $\mathrm{X}^{ \pm}$and $E^{*}=-1 / 6$ for $\mathrm{Y}^{ \pm}$. The energy can be written as

$$
E=E^{*}+E^{\prime},
$$

with small-amplitude energy perturbations

$$
E^{\prime}=\frac{p_{\theta}^{\prime 2}}{2}+5 p_{\phi}^{\prime 2}-\frac{1}{2}\left(E^{*}+\frac{1}{4}\right) \phi^{\prime 2}+\frac{1}{8}\left(\phi^{\prime}-2 \theta^{\prime}\right)^{2}
$$

that are quadratic in the perturbations.

A general solution can be constructed from eigenfunctions of the form

$$
\mathbf{x}^{\prime}(t)=\tilde{\mathbf{x}} e^{\lambda t},
$$

where $\lambda$ and $\tilde{\mathbf{x}}$ are constants. Substituting Eq. (73) into Eq. (69) yields $\lambda \tilde{\mathbf{x}}=A \tilde{\mathbf{x}}$, whence

$$
\left(\begin{array}{cccc}
-\lambda & 0 & 1 & 0 \\
0 & -\lambda & 0 & 10 \\
-1 & 1 / 2 & -\lambda & 0 \\
1 / 2 & E^{*} & 0 & -\lambda
\end{array}\right)\left(\begin{array}{c}
\tilde{\theta} \\
\tilde{\phi} \\
\tilde{p}_{\theta} \\
\tilde{p}_{\phi}
\end{array}\right)=0 .
$$

Setting the determinant of this $4 \times 4$ matrix to zero gives the eigenvalue condition

$$
\lambda^{4}+\left(1-10 E^{*}\right) \lambda^{2}-\left(\frac{5}{2}+10 E^{*}\right)=0 .
$$

Equation (74) determines the eigenvector

$$
\tilde{\mathbf{x}}=\left(\begin{array}{c}
\tilde{\theta} \\
\tilde{\phi} \\
\tilde{p}_{\theta} \\
\tilde{p}_{\phi}
\end{array}\right)=\left(\begin{array}{c}
1 \\
2\left(1+\lambda^{2}\right) \\
\lambda \\
\lambda\left(1+\lambda^{2}\right) / 5
\end{array}\right),
$$

whose overall amplitude is as yet undetermined; any multiple of Eq. (76) solves Eq. (74) as long as $\lambda$ satisfies Eq. (75).

It is often convenient to replace $\phi$ with $\beta=\phi-\phi_{m}$. For small perturbations, Eq. (42) yields

$$
\beta^{\prime}=\phi^{\prime}-\frac{3}{2} \theta^{\prime}
$$

for $\mathrm{X}^{ \pm}$and

$$
\beta^{\prime}=\phi^{\prime}-3 \theta^{\prime}
$$

for $\mathrm{Y}^{ \pm}$.

\section{B. Stability near $\mathbf{X}^{ \pm}$}

For $\mathrm{X}^{ \pm}$and $E^{*}=-1 / 3$, Eq. (75) gives two pairs of imaginary eigenvalues, $\lambda= \pm i \omega_{1}$ and $\lambda= \pm i \omega_{2}$, with $i=\sqrt{-1}$ and with frequencies $\omega_{1}$ and $\omega_{2}$ given by the positive roots of

$$
\begin{aligned}
& \omega_{1}^{2}=\frac{13-\sqrt{139}}{6}, \\
& \omega_{2}^{2}=\frac{13+\sqrt{139}}{6} .
\end{aligned}
$$

Equation (76) gives the associated complex eigenvectors

$$
\tilde{\mathbf{x}}_{j}^{ \pm}=\left(\begin{array}{c}
1 \\
2\left(1-\omega_{j}^{2}\right) \\
\pm i \omega_{j} \\
\pm i \omega_{j}\left(1-\omega_{j}^{2}\right) / 5
\end{array}\right)
$$


for $j=1$ and 2 , with $\tilde{\mathbf{x}}_{j}^{-}=\left(\tilde{\mathbf{x}}_{j}^{+}\right)^{*}$. Here, the \pm refers not to the sign of $\mathrm{X}^{ \pm}$but to the signs of the eigenvalues $\lambda= \pm i \omega_{j}$.

A linear superposition of the four eigenfunctions gives the general solution,

$$
\mathbf{x}^{\prime}(t)=\sum_{j=1}^{2}\left(C_{j}^{+} \tilde{\mathbf{x}}_{j}^{+} e^{+i \omega_{j} t}+C_{j}^{-} \tilde{\mathbf{x}}_{j}^{-} e^{-i \omega_{j} t}\right) .
$$

For this solution to be real, its complex coefficients must satisfy $C_{j}^{-}=\left(C_{j}^{+}\right)^{*}$. Accordingly, we write $C_{j}^{ \pm}=\left(3 C_{j} / 2\right) e^{ \pm i \delta_{j}}$, where $C_{j}$ and $\delta_{j}$ are real constants, and use these to rewrite Eq. (82) as

$$
\begin{aligned}
\left(\begin{array}{c}
\theta^{\prime}(t) \\
\phi^{\prime}(t)
\end{array}\right)= & C_{1}\left(\begin{array}{c}
3 \\
-7+\sqrt{139}
\end{array}\right) \cos \left(\omega_{1} t+\delta_{1}\right) \\
& +C_{2}\left(\begin{array}{c}
3 \\
-7-\sqrt{139}
\end{array}\right) \cos \left(\omega_{2} t+\delta_{2}\right) \\
\left(\begin{array}{c}
p_{\theta}^{\prime}(t) \\
p_{\phi}^{\prime}(t)
\end{array}\right)= & -C_{1} \omega_{1}\left(\begin{array}{c}
\frac{-7+\sqrt{139}}{10} \\
\frac{3}{3}
\end{array}\right) \sin \left(\omega_{1} t+\delta_{1}\right) \\
& -C_{2} \omega_{2}\left(\frac{-7-\sqrt{139}}{10}\right) \sin \left(\omega_{2} t+\delta_{2}\right) .
\end{aligned}
$$

Equations (83) and (84) give the general solution for $\mathbf{x}^{\prime}(t)$ near $\mathrm{X}^{ \pm}$, with four constants $\left(C_{1}, C_{2}, \delta_{1}\right.$, and $\left.\delta_{2}\right)$ that can be adjusted to satisfy arbitrary initial conditions on $\theta^{\prime}, \phi^{\prime}, p_{\theta}^{\prime}$, and $p_{\phi}^{\prime}$. The solution has two oscillatory normal modes with frequencies $\omega_{1}$ and $\omega_{2}$, amplitudes $C_{1}$ and $C_{2}$, and phases $\delta_{1}$ and $\delta_{2}$. Unless the initial conditions are chosen to isolate one of the normal modes, the behavior near the equilibrium positions $\mathrm{X}^{ \pm}$will be a linear combination of these two modes. Therefore, $\mathbf{x}_{\mathrm{X}^{ \pm}}^{*}$ are stable elliptical fixed points.

To isolate mode 1, we set $C_{2}=0$ in Eqs. (71), (72), (77), (83), and (84) to obtain

$$
\begin{gathered}
\theta^{\prime}(t)=\Theta_{1} \cos \left(\omega_{1} t+\delta_{1}\right), \\
\phi^{\prime}(t)=\Phi_{1} \cos \left(\omega_{1} t+\delta_{1}\right), \\
\beta^{\prime}(t)=\left(-\frac{23}{2}+\sqrt{139}\right) C_{1} \cos \left(\omega_{1} t+\delta_{1}\right), \\
\frac{\beta^{\prime}(t)}{\theta^{\prime}(t)}=\frac{1}{3}\left(-\frac{23}{2}+\sqrt{139}\right)=0.097, \\
C_{1}^{2}=6 \frac{E+1 / 3}{278-23 \sqrt{139}}, \\
\Theta_{1}=3 C_{1}, \\
\Phi_{1}=(-7+\sqrt{139}) C_{1},
\end{gathered}
$$

where $\Theta_{1}$ and $\Phi_{1}$ are the amplitudes of $\theta^{\prime}(t)$ and $\phi^{\prime}(t)$, respectively. Equation (88) states that the variations in $\beta(t)$ are small compared with variations in $\theta(t)$, and the magnetic moment of the free sphere therefore stays closely aligned with the local magnetic field. According to $U=-B \cos \beta$ [Eq. (45)], the free sphere must travel to weaker field regions to reach its maximum potential energy $U=E$, where $\theta$ and $\phi$ reach their maximum values, $\Theta_{1}$ and $\Phi_{1}$, and the kinetic energy vanishes. We therefore refer to this mode as the "traveling mode." In this mode, $\theta^{\prime}(t), \phi^{\prime}(t)$, and $\beta^{\prime}(t)$ share the same sign and oscillate in phase with each other.

To isolate mode 2, we set $C_{1}=0$ in Eqs. (71), (72), (77), (83), and (84) to obtain

$$
\begin{gathered}
\theta^{\prime}(t)=\Theta_{2} \cos \left(\omega_{2} t+\delta_{2}\right), \\
\phi^{\prime}(t)=-\Phi_{2} \cos \left(\omega_{2} t+\delta_{2}\right), \\
\beta^{\prime}(t)=-\left(\frac{23}{2}+\sqrt{139}\right) C_{2} \cos \left(\omega_{2} t+\delta_{2}\right), \\
\frac{\beta^{\prime}(t)}{\theta^{\prime}(t)}=-\frac{1}{3}\left(\frac{23}{2}+\sqrt{139}\right)=-7.8, \\
C_{2}^{2}=6 \frac{E+1 / 3}{278+23 \sqrt{139}}, \\
\Theta_{2}=3 C_{2}, \\
\Phi_{2}=(7+\sqrt{139}) C_{2} .
\end{gathered}
$$

Equation (95) states that the variations in $\beta(t)$ are large compared with variations in $\theta(t)$, and the direction of the magnetic moment of the free sphere therefore departs significantly from the direction of the local magnetic field. According to $U=-B \cos \beta[\mathrm{Eq}$. (45)], the free sphere need not travel large distances to weaker field regions to reach its maximum potential energy $U=E$ because it can achieve this value primarily by rotating in place. We therefore refer to this mode as the "rotating mode." In this mode, $\phi^{\prime}(t)$ and $\beta^{\prime}(t)$ are in phase with each other and are out of phase with $\theta^{\prime}(t)$. The rotating mode has a frequency $\omega_{2}$ that is about 4.5 times larger than the frequency $\omega_{1}$ of the traveling mode.

\section{Stability near $\mathbf{Y}^{ \pm}$}

For $\mathrm{Y}^{ \pm}$and $E^{*}=-1 / 6$, Eq. (75) gives a pair $\lambda= \pm i \omega_{3}$ of imaginary eigenvalues and a pair $\lambda= \pm \sigma_{4}$ of real ones, with frequency $\omega_{3}$ and growth rate growth rate $\sigma_{4}$ given by the positive roots of

$$
\begin{gathered}
\omega_{3}^{2}=\frac{8+\sqrt{94}}{6}, \\
\sigma_{4}^{2}=\frac{-8+\sqrt{94}}{6} .
\end{gathered}
$$

Equation (76) gives the associated eigenvectors

$$
\tilde{\mathbf{x}}_{3}^{ \pm}=\left(\begin{array}{c}
1 \\
2\left(1-\omega_{3}^{2}\right) \\
\pm i \omega_{3} \\
\pm i \omega_{3}\left(1-\omega_{3}^{2}\right) / 5
\end{array}\right)
$$




$$
\tilde{\mathbf{x}}_{4}^{ \pm}=\left(\begin{array}{c}
1 \\
2\left(1+\sigma_{4}^{2}\right) \\
\pm \sigma_{4} \\
\pm \sigma_{4}\left(1+\sigma_{4}^{2}\right) / 5
\end{array}\right),
$$

with $\tilde{\mathbf{x}}_{3}^{-}=\left(\tilde{\mathbf{x}}_{3}^{+}\right)^{*}$.

A linear superposition of the four eigenfunctions gives the general solution

$$
\begin{aligned}
\mathbf{x}^{\prime}(t)= & C_{3}^{+} \tilde{\mathbf{x}}_{3}^{+} e^{+i \omega_{3} t}+C_{3}^{-} \tilde{\mathbf{x}}_{3}^{-} e^{-i \omega_{3} t} \\
& +3 C_{4}^{+} \tilde{\mathbf{x}}_{4}^{+} e^{+\sigma_{4} t}+3 C_{4}^{-} \tilde{\mathbf{x}}_{4}^{-} e^{-\sigma_{4} t}
\end{aligned}
$$

Writing $C_{3}^{ \pm}=\left(3 C_{3} / 2\right) e^{ \pm i \delta_{3}}$ allows us to write Eq. (103) as

$$
\begin{aligned}
\left(\begin{array}{l}
\theta^{\prime}(t) \\
\phi^{\prime}(t)
\end{array}\right)= & C_{3}\left(\begin{array}{c}
3 \\
-2-\sqrt{94}
\end{array}\right) \cos \left(\omega_{3} t+\delta_{3}\right) \\
& +\left(\begin{array}{c}
3 \\
-2+\sqrt{94}
\end{array}\right)\left(C_{4}^{+} e^{+\sigma_{4} t}+C_{4}^{-} e^{-\sigma_{4} t}\right), \quad(104) \\
\left(\begin{array}{c}
p_{\theta}^{\prime}(t) \\
p_{\phi}^{\prime}(t)
\end{array}\right)= & -C_{3} \omega_{3}\left(\frac{-2-\sqrt{94}}{10}\right) \sin \left(\omega_{3} t+\delta_{3}\right) \\
& \times \sigma_{4}\left(\frac{-2+\sqrt{94}}{10}\right)\left(C_{4}^{+} e^{+\sigma_{4} t}-C_{4}^{-} e^{-\sigma_{4} t}\right) .
\end{aligned}
$$

Equations (104) and (105) give the general solution for $\mathbf{x}^{\prime}(t)$ near $\mathrm{Y}^{ \pm}$, with four constants $\left(C_{3}, \delta_{3}, C_{4}^{+}\right.$, and $\left.C_{4}^{-}\right)$that can be adjusted to satisfy arbitrary initial conditions on $\theta^{\prime}, \phi^{\prime}, p_{\theta}^{\prime}$, and $p_{\phi}^{\prime}$. The solution has a stable elliptical mode with frequency $\omega_{3}$ and an unstable hyperbolic mode with growth rate $\sigma_{4}$. If $C_{4}^{+} \neq 0$, the unstable mode dominates at long times, driving the phase-space trajectory far from the fixed point. The fixed points $\mathbf{x}_{\mathrm{Y}^{ \pm}}^{*}$ are therefore unstable hyperbolic fixed points, with the elliptical mode providing oscillations as the phase-space trajectory departs from the fixed point.

To isolate the oscillatory mode, we set $C_{4}^{ \pm}=0$ in Eqs. (71), (72), (78), (104), and (105) to obtain

$$
\begin{gathered}
\theta^{\prime}(t)=\Theta_{3} \cos \left(\omega_{3} t+\delta_{3}\right), \\
\phi^{\prime}(t)=-\Phi_{3} \cos \left(\omega_{3} t+\delta_{3}\right), \\
\beta^{\prime}(t)=-(11+\sqrt{94}) C_{3} \cos \left(\omega_{3} t+\delta_{3}\right), \\
\frac{\beta^{\prime}(t)}{\theta^{\prime}(t)}=-\frac{1}{3}(11+\sqrt{94})=-6.9, \\
C_{3}^{2}=\frac{1+6 E}{94+11 \sqrt{94}}, \\
\Theta_{3}=3 C_{3}, \\
\Phi_{3}=(2+\sqrt{94}) C_{3} .
\end{gathered}
$$

Like Eq. (95), Eq. (109) gives variations in $\beta(t)$ that are large compared with, and out of phase with, variations in $\theta(t)$. Accordingly, the magnetic moment of the free sphere departs significantly from the direction of the local magnetic field, and the free sphere primarily rotates in place rather than translating significant distances. Since the hyperbolic mode renders this oscillating mode unstable for general initial conditions, we refer to it as the "unstable rotating mode."

To isolate the unstable component of the hyperbolic mode, we set $C_{3}=C_{4}^{-}=0$ in Eqs. (104) and (105) to yield

$$
\mathbf{x}^{\prime}(t)=C_{4}^{+}\left(\begin{array}{c}
3 \\
-2+\sqrt{94} \\
3 \sigma_{4} \\
\frac{\sigma_{4}}{10}(-2+\sqrt{94})
\end{array}\right) e^{+\sigma_{4} t} .
$$

For this solution, perturbations grow with time and the trajectory departs exponentially from the fixed point. Setting $C_{3}=C_{4}^{+}=0$ gives the stable component

$$
\mathbf{x}^{\prime}(t)=C_{4}^{-}\left(\begin{array}{c}
3 \\
-2+\sqrt{94} \\
-3 \sigma_{4} \\
-\frac{\sigma_{4}}{10}(-2+\sqrt{94})
\end{array}\right) e^{-\sigma_{4} t}
$$

for which perturbations decay with time and the trajectory approaches the fixed point exponentially. Since both components intersect the fixed point and since $E$ is conserved along phase-space trajectories, these two components lie on a separatrix of energy $E=-1 / 6$ that serves as a boundary between different behaviors in phase space, with Eqs. (113) and (114), respectively, representing the unstable and stable manifolds of the fixed point. ${ }^{22}$

Substituting Eq. (113) or Eq. (114) into Eqs. (71) and (72) yields $E=-1 / 6$ identically; all terms involving $C_{4}^{ \pm}$cancel each other, and the unstable and stable manifolds exist only for $E=-1 / 6$. This is in contrast to the oscillatory modes, which admit relationships between their coefficients and the energy given by Eqs. (89), (96), and (110). The coefficients $C_{4}^{ \pm}$are determined by the initial conditions alone.

\section{FINITE-AMPLITUDE PERIODIC MODES}

\section{A. General}

Having identified small-amplitude periodic sliding modes near $\mathrm{X}^{ \pm}$and $\mathrm{Y}^{ \pm}$, we now ask whether these modes persist to finite amplitudes, and ask whether these modes are joined by other periodic modes as the energy increases.

We consider modes with period $T$ and angular frequency $\omega=2 \pi / T$ satisfying the initial $(t=0)$ and final $(t=T)$ conditions

$$
\begin{gathered}
\theta(0)=\theta^{*} \quad \theta(T)=\theta^{*}+2 \pi n, \\
\phi(0)=\phi^{*} \quad \phi(T)=\phi^{*}+4 \pi n, \\
p_{\theta}(0)=p_{\theta}(T), \\
p_{\phi}(0)=p_{\phi}(T),
\end{gathered}
$$


where $\theta^{*}$ and $\phi^{*}$ correspond to one of the equilibrium positions, $\mathrm{X}^{ \pm}$or $\mathrm{Y}^{ \pm}$, and $n=0, \pm 1$. Equations (115) and (116) place the free sphere at one of the equilibrium positions at $t=0$, and return this sphere to this same position at time $t=T$. Equation (52) therefore requires

$$
U(0)=U(T)=E^{*}=-1 / 3
$$

for $\mathrm{X}^{ \pm}$and

$$
U(0)=U(T)=E^{*}=-1 / 6
$$

for $\mathrm{Y}^{ \pm}$. The value $n=0$ corresponds to oscillations of the free sphere about the equilibrium position, while $n= \pm 1$ corresponds to the free sphere making one complete orbit around the fixed sphere, in the counterclockwise direction for $n=+1$ and the clockwise direction for $n=-1$. We have not considered periodic modes making more than one orbit (with $|n|>1$ ). The final conditions on $\theta$ and $\phi$ differ because $\phi$ goes through two cycles for each cycle of $\theta$. Equations (117) and (118) demand that the final momenta equal the initial momenta, ensuring a periodic solution. The small-amplitude oscillatory modes of Eqs. (85)-(91), (92)-(98), and (106)-(112) satisfy Eqs. (115)-(118) for $\delta_{1}=\delta_{2}=\delta_{3}=-\pi / 2$.

Bounds on the initial momenta may be obtained by evaluating Eq. (51) at $t=0$, giving

$$
E=\frac{p_{\theta}^{2}(0)}{2}+5 p_{\phi}^{2}(0)+E^{*} .
$$

Setting $p_{\theta}(0)=0$ and solving for $p_{\phi}(0)$ gives its bounding values $p_{\phi}(0)= \pm p_{\phi}^{\mathrm{m}}(0)$, where

$$
p_{\phi}^{\mathrm{m}}(0)=\sqrt{\frac{E-E^{*}}{5}}
$$

is the maximum initial value of $p_{\phi}$. For a particular choice of $p_{\phi}(0)$ satisfying

$$
-p_{\phi}^{\mathrm{m}}(0) \leq p_{\phi}(0) \leq p_{\phi}^{\mathrm{m}}(0),
$$

and for a particular energy $E$, Eq. (121) gives

$$
p_{\theta}(0)=\sqrt{2\left[E-E^{*}-5 p_{\phi}^{2}(0)\right]} .
$$

We use the positive square root in Eq. (124) without loss of generality. Both in-phase and out-of-phase periodic modes may be realized in combination with the full range of values of $p_{\phi}(0)$ given by Eq. (123).

We seek periodic solutions at a particular value of $E$ by finding values of $\phi(t)$ at equally spaced values of $p_{\phi}(0)$ over the full range of Eq. (123), with spacing $\Delta p_{\phi}=0.0002$. For each value of $p_{\phi}(0)$, we obtain $p_{\theta}(0)$ from Eq. (124) and use fourth-order Runge Kutta to integrate Eqs. (53)-(56) with a fixed time step $\Delta t=0.0001$, subject to the initial and final conditions given by Eqs. (115)-(118). ${ }^{23}$ We integrate until we reach a time $t$ at which $\Delta \theta=\theta(t)-\theta^{*}-2 \pi n$ changes sign, and at which $p_{\theta}$ has the same sign as it had initially, for $n=0, \pm 1$. In order to limit computational time lost on fruitless searches for solutions, we abandon the search if $t$ reaches 50 without these conditions being satisfied, acknowledging that this restriction excludes high-period solutions. A linear interpolation between the time step at which $\Delta \theta$ changes sign and the previous time step increases the accuracy of our estimate for $t$, and gives the corresponding value of $\Delta \phi=\phi(t)-\phi^{*}-4 \pi n$.

Having thus obtained a value of $\Delta \phi$ for each value of $p_{\phi}(0)$, we use Brent's method to look for roots of $\Delta \phi$ vs. $p_{\phi}(0)$ bracketed by pairs of values for which $\Delta \phi$ changes sign. ${ }^{24}$ In this way, we identify initial conditions $p_{\theta}(0)$ and $p_{\phi}(0)$ that give solutions that are periodic in $\theta$ and $\phi$, with $\theta(0)=\theta^{*}$ and $\phi(0)=\phi^{*}$. Finally, we accept a solution as fully periodic, with period $t=T$ and frequency $\omega=2 \pi / T$, only if $\left|p_{\theta}(T)-p_{\theta}(0)\right|<0.0001$ and $\left|p_{\phi}(T)-p_{\phi}(0)\right|<0.0001$.

The number of periodic solutions and the requisite computing time increase with decreasing $\Delta p_{\phi}$, that is, finer resolution of $\phi(t)$ yields more roots and requires longer computing times. We used $\Delta p_{\phi}=0.0002$ to obtain a significant number of solutions - up to 26 solutions for each value of $E$-in a reasonable time.

We seek for solutions for a full range of values of $E>E^{*}$, at equally spaced values separated by $\Delta E=0.0005$. While solutions with $E<0$ satisfy Eq. (47), solutions with $E>0$ may violate this condition, which ensures continued contact between the spheres. For $U>0$, the repulsive magnetic force violates Eq. (47) and drives the spheres apart regardless of the value of $p_{\theta}$. For $U<0$, large $p_{\theta}$ implies a large centrifugal force that violates Eq. (47) and drives the spheres apart. Violation of this condition causes the free sphere to lose contact with the fixed sphere, at least momentarily. To ensure periodic solutions with constant contact between the spheres, we accept only those solutions that obey Eq. (47) at all times. This sets an upper limit on the energy of periodic sliding solutions, which turns out to be $E=0.191$.

For oscillatory modes, we define the amplitude $\Theta$ as the maximum value of $\left|\theta(t)-\theta^{*}\right|$ reached during one period $T$, and define the amplitude $\Phi$ as the maximum value of $\left|\phi(t)-\phi^{*}\right|$.

During the coding and debugging process, we used MagPhyx software to visualize some of these solutions and to verify their periodicity. ${ }^{25}$

\section{B. Initial position $\mathbf{X}^{ \pm}$}

We first investigate finite-amplitude modes with initial positions $\theta(0)=0$ and $\phi(0)=0$ corresponding to the equilibrium position $\mathrm{X}^{+}$, noting that $\mathrm{X}^{-}$gives the same modes through the symmetry transformation $\theta \rightarrow \theta+\pi$. Following the procedures outlined in Sec. VIII A, we found 4548 oscillatory solutions and 4513 orbital solutions using a FORTRAN code that ran for 28 hours on a $2.6 \mathrm{GHz}$ MacBook Pro. Each solution is shown as a small filled circle in Fig. 5, where adjacent circles generally merge to form continuous traces.

The two periodic oscillatory modes that appear in Fig. 5 at $E=-1 / 3$ correspond to the small-amplitude traveling and rotating modes of Sec. VII B, modes 1 and 2. The frequencies 
of these modes decrease with increasing energy (Fig. 6), and their amplitudes increase with increasing energy (Fig. 7), as might be expected. As shown in Fig. 8, mode 1 makes large excursions in $\theta$ that are in phase with its small excursions in $\beta$, and departs slightly from the small-amplitude relationship between $\beta$ and $\theta$ given by Eq. (87) except at small amplitudes. Mode 2 makes large excursions in $\beta$ that are out of phase with its small excursions in $\theta$, and honors the small-amplitude relationship given by Eq. (94) at finite amplitudes. MagPhyx Demos 11 and 12, respectively, visualize modes 1 and 2 for $E=-0.29 .{ }^{25}$

At $E=-0.236$, mode 1 spawns two new oscillatory modes, labeled $5^{ \pm}$in Fig. 5. Each adds 5 rotation cycles to the traveling motion of mode 1 . Mode $5^{+}$has smaller $\Theta$ and larger $\Phi$ than mode $5^{-}$, and the two modes are $180^{\circ}$ out of


FIG. 5. Initial values of the spin angular momentum, $p_{\phi}(0)$, vs. energy $E$ for finite-amplitude periodic solutions of Eqs. (53)-(56) satisfying Eqs. (115)-(118) with the free sphere located initially at $\mathrm{X}^{ \pm}$(small filled circles merging to form continuous traces), with periodic modes at energies $E=-0.29,-0.212,-0.18,-0.167,-0.166,-0.01,0.06$, and 0.191 identified by open circles. Dashed lines give the bounding values $p_{\phi}(0)=$ $\pm p_{\phi}^{\mathrm{m}}(0)$ given by Eq. (122). Primed numerical labels identify orbital modes (green) and unprimed labels identify oscillatory modes (red). Frame (b) shows a closeup of the region outlined by the square in frame (a). phase [Figs. 7 and Fig. 8(b)]. MagPhyx Demo 13 visualizes mode $5^{+}$for $E=-0.212 .{ }^{25}$ The frequency of mode $5^{-}$ exceeds that of mode $5^{+}$by about $0.1 \%$, a difference that is not apparent in Fig. 6, where the two frequencies collapse onto a single trace, labeled "5." Mode $5^{-}$expires at $E=0$, where it merges with mode 2 . Mode $5^{+}$expires at about $E=-0.05$, where it merges with $p_{\phi}(0)=p_{\phi}^{\mathrm{m}}(0)$. The left side of Fig. 5(b) shows that the bifurcation at $E=-0.236$ is "soft," meaning that, as $E$ increases, mode 1 gradually turns into mode $5^{-}$in preparation for the actual bifurcation, which occurs where mode 1 is reborn and mode $5^{+}$appears. Plots of $\beta$ vs. $\theta$ for modes 1 and $5^{ \pm}$are shown in Fig. 8(b).

At $E=-0.187$, mode 1 spawns two more oscillatory modes, labeled $6^{ \pm}$in Fig. 5. These are in fact the same mode, with mode $6^{-}$following the same trajectory as mode $6^{+}$, but in reverse [Fig. 8(c)]. These modes add 6 rotation cycles to the traveling motion of mode 1 . Their independently computed frequencies and amplitudes agree to one part in $10^{7}$. Figure 5(b) shows that the bifurcation at $E=-0.187$ is "sharp," meaning that $6^{+}$and $6^{-}$appear abruptly, at the same

(a)

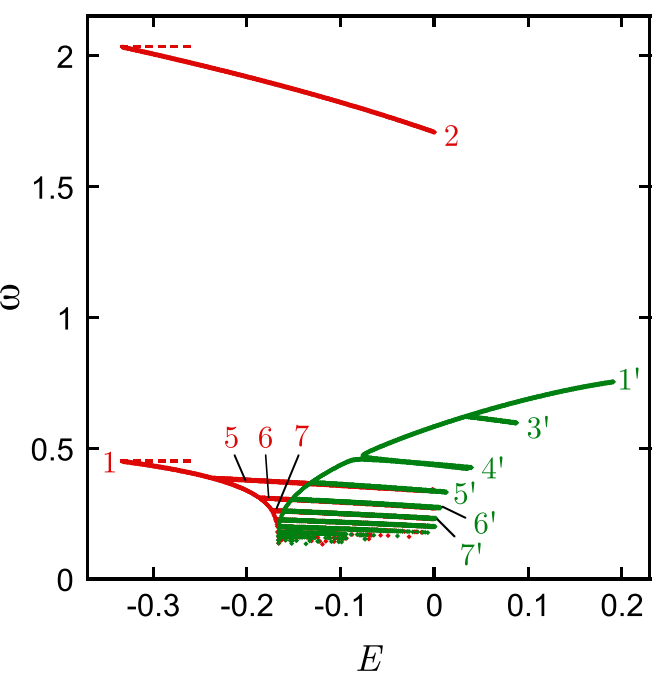

(b)

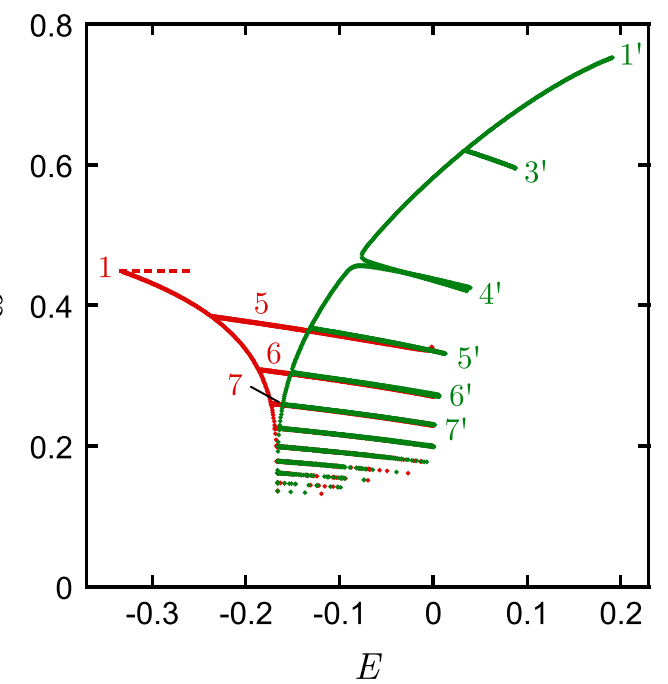

FIG. 6. Frequencies of the finite-amplitude periodic modes of Fig. 5, with detail of the lower portion of frame (a) shown in frame (b). Horizontal dashed lines give the small-amplitude frequencies given by Eqs. (79) and (80). 
(a)

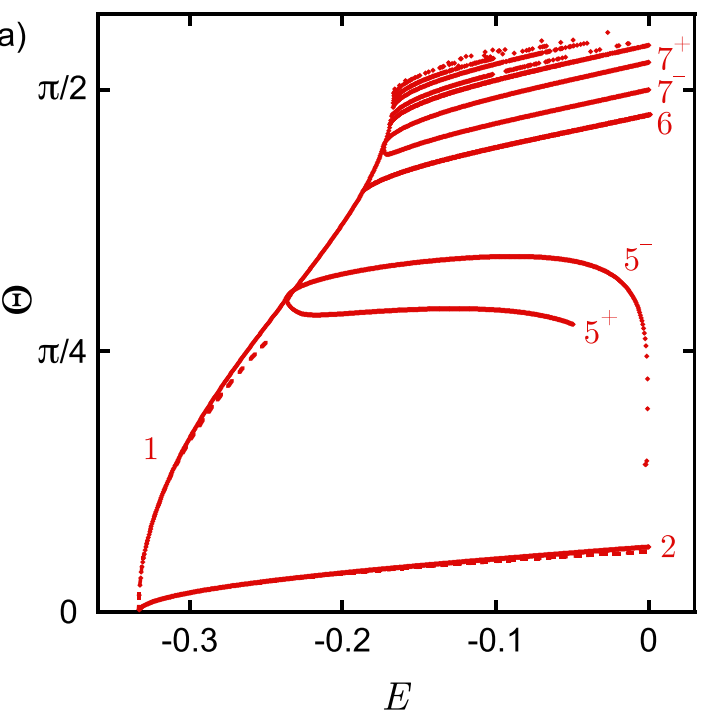

(b)

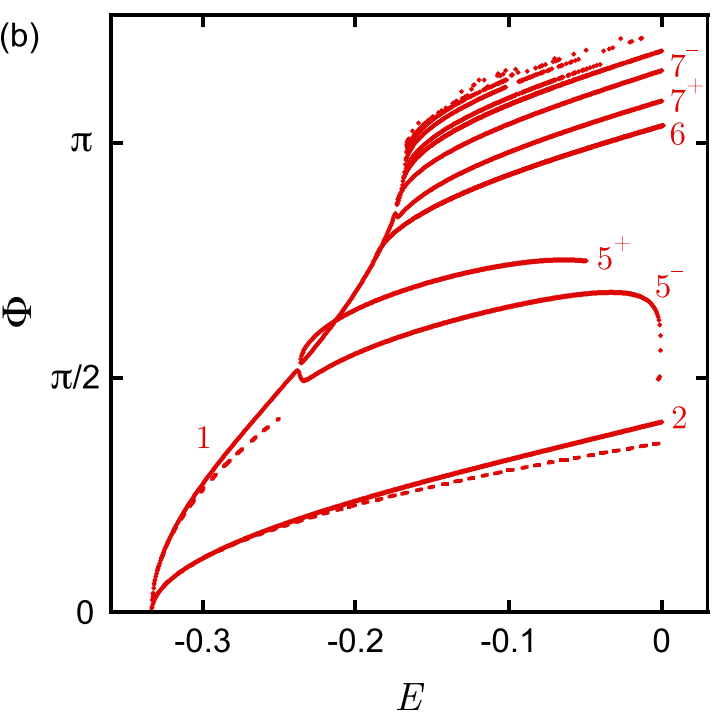

FIG. 7. Amplitudes of finite-amplitude oscillatory modes as a function of the energy $E$ (solid traces). Frame (a) shows the amplitude $\Theta$ of $\theta(t)$, and frame (b) the amplitude $\Phi$ of $\phi(t)$. Also shown are the amplitudes of smallamplitude oscillations given by Eqs. (89)-(91) and (96)-(98) (dashed traces).

value of the energy. MagPhyx Demo 14 visualizes mode $6^{+}$ for $E=-0.18 .^{25}$

Mode 1 continues to spawn oscillatory modes as $E$ approaches $E_{c}=-1 / 6$ from below, with soft bifurcations for odd-numbered modes and sharp bifurcations for evennumbered modes. Fig. 9 shows bifurcation values of the frequency ratio $\omega_{2} / \omega_{1}$ and the energy difference $E_{c}-E$ vs. mode number $n$ for $n$-cycle bifurcations approaching $E_{c}$. Evidently, mode $n^{ \pm}$is spawned only after $\omega_{2} / \omega_{1}$ exceeds $n$, indicating that mode 2 is responsible for the $n$ rotation cycles that are added to the traveling motion of mode 1 . The smallest number of rotation cycles is $n=5$, consistent with $\omega_{2} /$ $\omega_{1} \geq 4$.5. Figure 6(b) shows a precipitous drop in $\omega_{1}$ vs. $E$ at $E=E_{c}$, and hints of an infinite sequence of bifurcations with $\omega_{1} \rightarrow 0$ as $E \rightarrow E_{c}$. Indeed, Fig. 9 shows bifurcations up to $n=14$, which has bifurcation values $E_{c}-E=5.840 \times 10^{-6}$ (a)



(b)

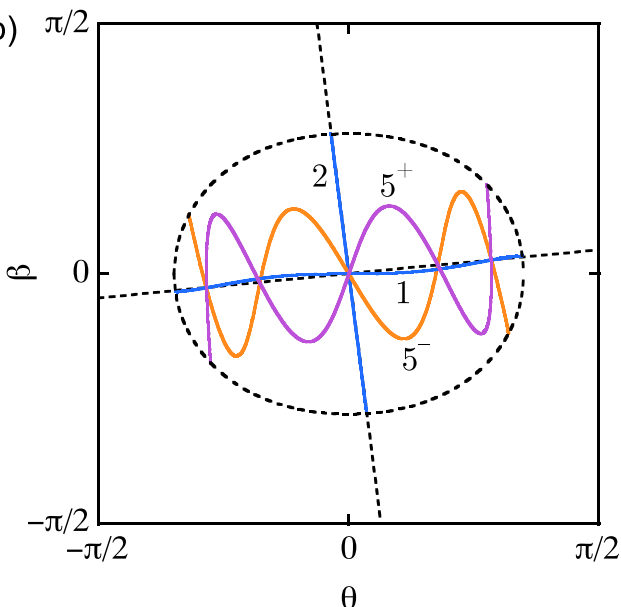

(c)

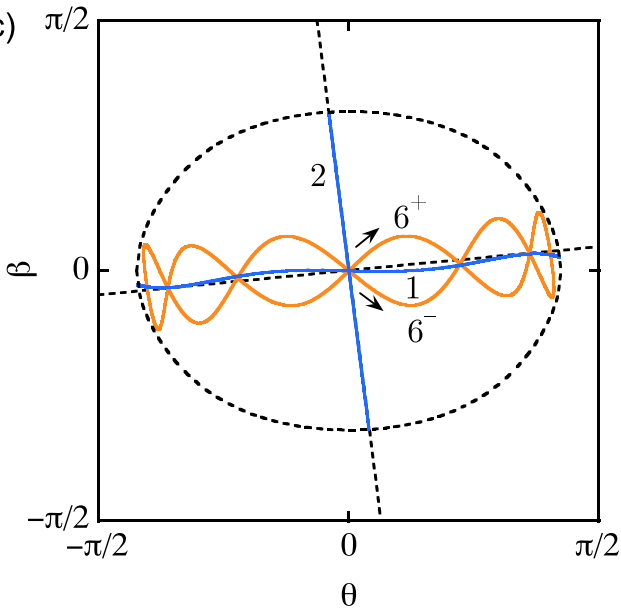

FIG. 8. Plots of $\beta$ vs. $\theta$ for finite-amplitude oscillations with energies $E=-0.29$ (a), -0.212 (b), and -0.18 (c) (solid traces). Oval dashed traces give the domain boundaries of Eq. (50), and straight dashed traces give the relationships of Eqs. (88) and (95) for small-amplitude oscillations. The arrows in (c) show the initial directions of motion for modes $6^{+}$and $6^{-}$.

and $\omega_{2} / \omega_{1}=14.99$. To obtain the values shown in this figure, we increased the energy resolution of Sec. VIII A by dividing $\Delta E$ by factors of 10 until $E_{c}-E$ and $\omega_{2} / \omega_{1}$ changed by less than $0.1 \%$. For $n=14$, this process required $\Delta E=1 \times 10^{-9}$. Although we ended our search for bifurcations at $n=14$, we have no reason to think that bifurcations stop there, and 


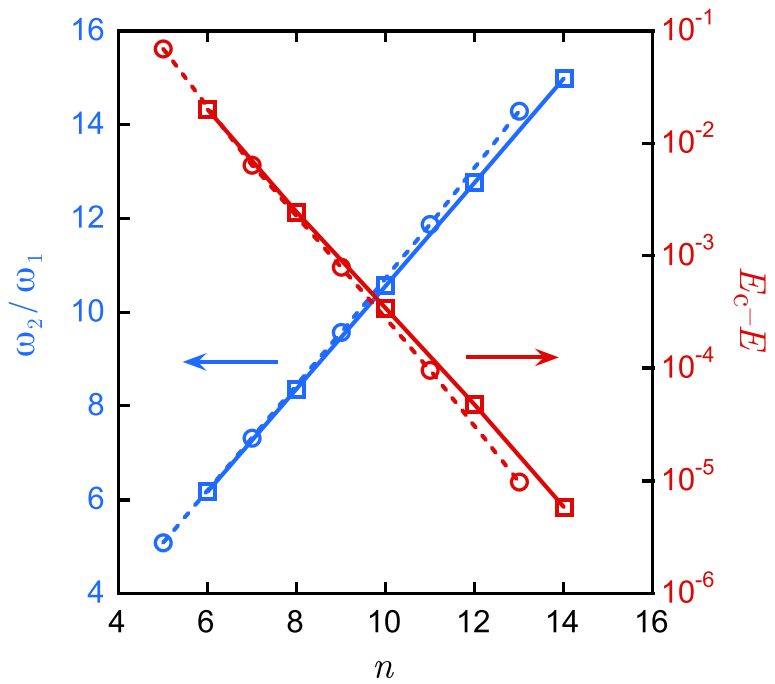

FIG. 9. Frequency ratios $\omega_{2} / \omega_{1}$ and energy differences $E_{c}-E$ vs. mode number $n$ for $n$-cycle bifurcations approaching the value $E_{c}=-1 / 6$ from below. Squares give results for even $n$, with solid traces joining these results to guide the eye, while circles and dashed traces give results for odd $n$.

(a)

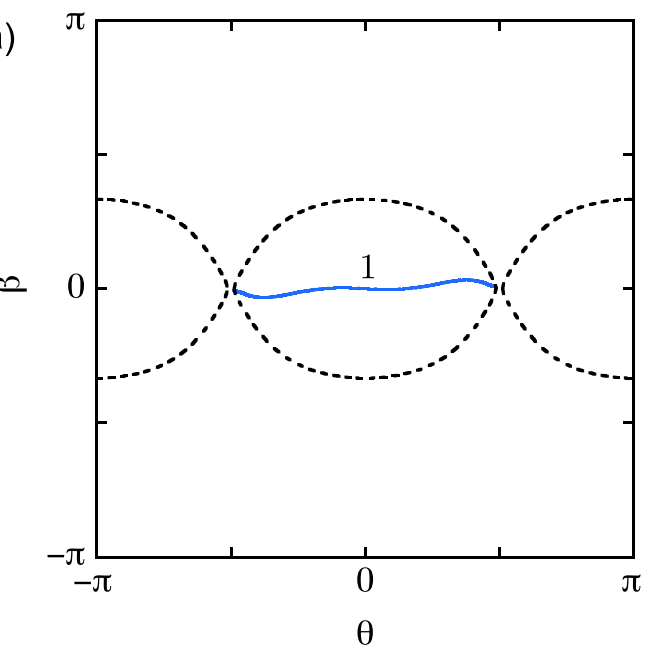

(b)

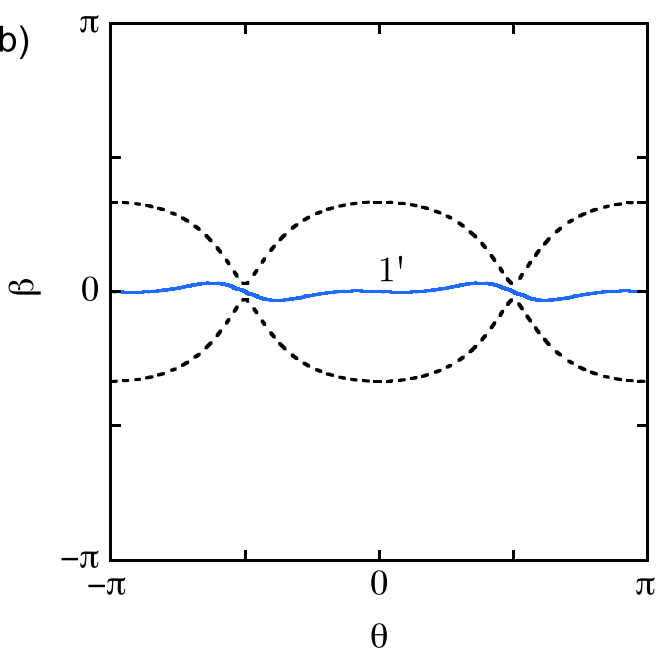

FIG. 10. Plots of $\beta$ vs. $\theta$ (solid traces) and domain boundaries (dashed traces). Panel (a) shows oscillatory mode 1 with $E=-0.167$ and a discontiguous domain. Panel (b) shows orbital mode $1^{\prime}$ with $E=-0.166$ and a contiguous domain.
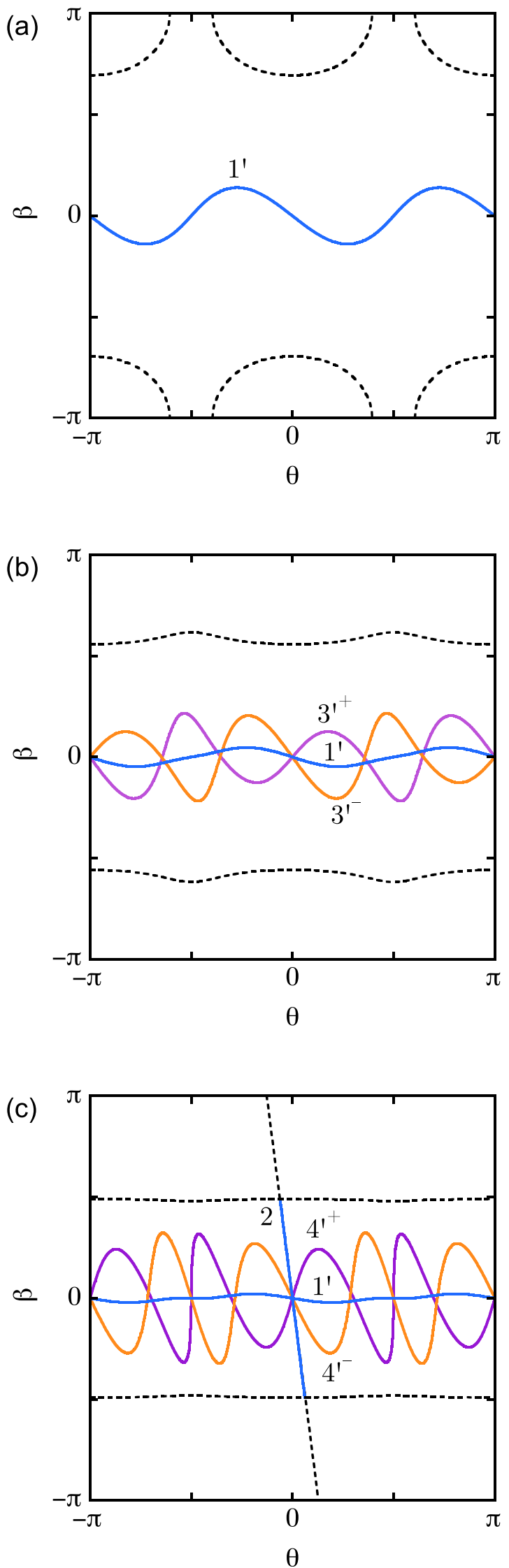

FIG. 11. Plots of $\beta$ vs. $\theta$ for periodic modes with energies $E=0.191$ (a), 0.06 (b), and -0.01 (c) (solid traces). In frame (c), the nearly vertical dashed trace gives the relationship of Eq. (95) for small-amplitude oscillations, and the solid trace that overlays it gives mode 2 . The other dashed traces in frames (a)-(c) give the domain boundaries of Eq. (50). 
ample reason to suspect that they cascade indefinitely to the cumulation point at $E=E_{c}$.

Figure 10(a) and MagPhyx Demo 15 show mode 1 for $E=-0.167$, just below $E_{c}=-1 / 6 .^{25}$ For $E<E_{c}$, mode 1 is oscillatory, the free sphere being confined to one of the two domains shown in Figs. 3 and 4. For $E>E_{c}$, these domains merge and mode 1 describes the free sphere orbiting completely around the fixed sphere. We designate this orbital mode as mode $1^{\prime}$ to distinguish it from its oscillatory brother, and use primes below to name other orbital modes. Figure 10(b) and MagPhyx Demo 16 show mode $1^{\prime}$ for $E=-0.166$, just above $E_{c}=-1 / 6$. $^{25}$

For $E_{c}<E<0$, Fig. 5 shows both oscillatory and orbital modes, and for $E>0$, only orbital modes. The highest-energy
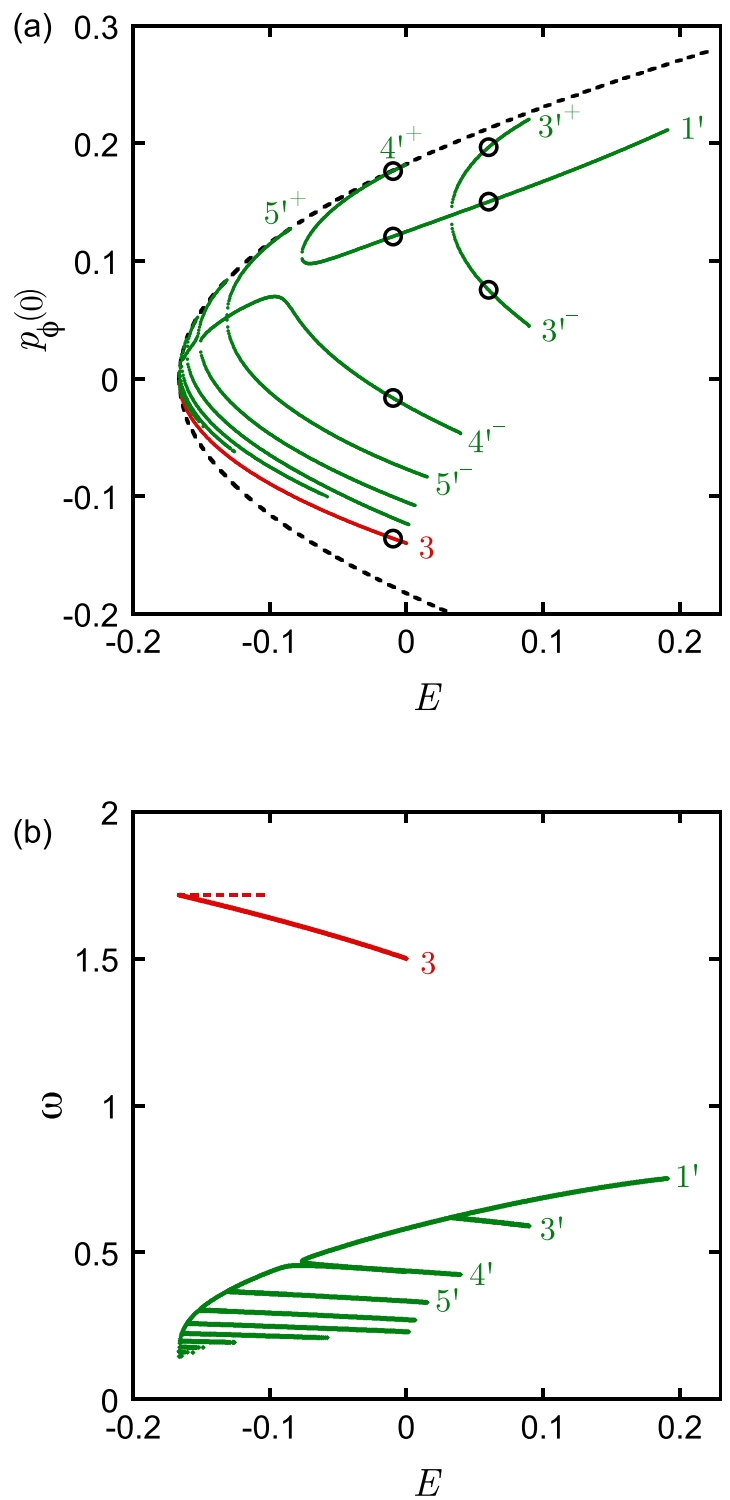

FIG. 12. (a) Initial values of the spin angular momentum, $p_{\phi}(0)$, vs. energy $E$ for finite-amplitude periodic solutions of Eqs. (53)-(56) satisfying Eqs. (115)-(118) with the free sphere located initially at $\mathrm{Y}^{ \pm}$(small filled circles merging to form continuous traces), with periodic modes at energies $E=-0.06$ and 0.01 identified by open circles. Dashed lines give the bounding values $p_{\phi}(0)= \pm p_{\phi}^{\mathrm{m}}(0)$ given by Eq. (122). (b) Frequencies of the finite-amplitude periodic modes, with the horizontal dashed line giving the small-amplitude frequency $\omega_{3}$ of Eq. (99). orbital mode is mode $1^{\prime}$, at energy $E=0.191$ [Fig. 11(a), MagPhyx Demo 20]. [25] Moving to lower energies, mode $1^{\prime}$ spawns modes $3^{\prime \pm}$ with three rotation cycles [Fig. 11(b), with mode $3^{\prime+}$ visualized at $E=0.06$ in MagPhyx Demo 19], ${ }^{25}$ modes $4^{\prime \pm}$ with four rotation cycles [Fig. 11(c), with mode $4^{\prime+}$ visualized at $E=-0.01$ in MagPhyx Demo 18], ${ }^{25}$ and so on, possibly culminating in an infinite number of cycles at $E=E_{c}$ as before (Fig. 11). In contrast to oscillatory bifurcations, orbital bifurcations are sharp for odd-numbered modes and soft for even-numbered modes.

As seen in Fig. 5, mode 2 persists, without bifurcating, to $E=0$, above which the free sphere loses contact with the fixed sphere. Figure 11(c) and MagPhyx Demo 17 show mode 2 at $E=-0.01 .^{25}$

\section{Initial position $\mathbf{Y}^{ \pm}$}

We now investigate finite-amplitude modes with initial positions $\theta(0)=\pi / 2$ and $\phi(0)=\pi$ corresponding to the equilibrium position $\mathrm{Y}^{+}$, noting that $\mathrm{Y}^{-}$gives the same modes through the symmetry transformation $\theta \rightarrow \theta+\pi$. Following the procedures outlined in Sec. VIII A, we found 334 oscillatory solutions and 2755 orbital solutions using a FORTRAN code that ran for 12 hours on a $2.6 \mathrm{GHz}$ MacBook Pro. Each solution is shown as a small filled circle in Fig. 12, where adjacent circles generally merge to form continuous traces.

Figure 12 shows a family of orbital modes for $-1 / 6$ $<E<0.191$ and one oscillatory mode (mode 3 ) for $-1 / 6$ $<E<0$, whose amplitudes are shown in Fig. 13. Figure 14 shows $\beta$ vs. $\theta$ plots for oscillatory mode 3 and for orbital modes $1^{\prime}, 3^{\prime \pm}$, and $4^{\prime \pm}$. MagPhyx Demos 21, 22, and 23, respectively, visualize mode $3^{\prime+}$ at $E=0.06$, mode 3 at $E=-0.01$, and mode $4^{\prime+}$ at $E=-0.01$.

While modes $1^{\prime}$ and $4^{\prime \pm}$ of Fig. 14 are identical to modes $1^{\prime}$ and $4^{\prime \pm}$ of Fig. 11 , mode $3^{\prime \pm}$ is different. The reason is that mode $3^{\prime \pm}$ of Fig. 14 has an antinode at $\theta=0$, and is therefore incompatible with the initial condition $\phi(0)=0$

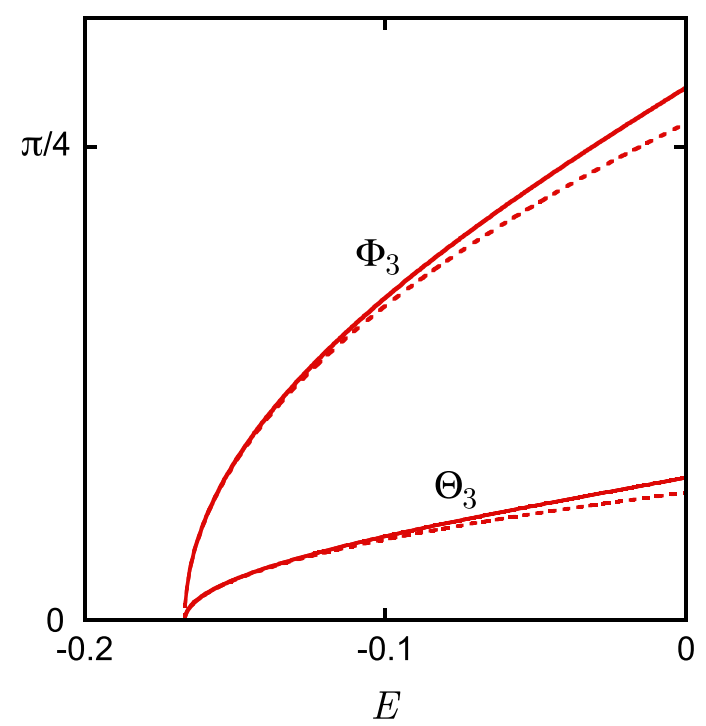

FIG. 13. Amplitudes $\Theta_{3}$ and $\Phi_{3}$ of finite-amplitude oscillatory mode 3 as a function of the energy $E$ for the free sphere located initially at $\mathrm{Y}^{ \pm}$(solid traces). Also shown are the amplitudes of small-amplitude oscillations given by Eqs. (110)-(112) (dashed traces). 
(a)

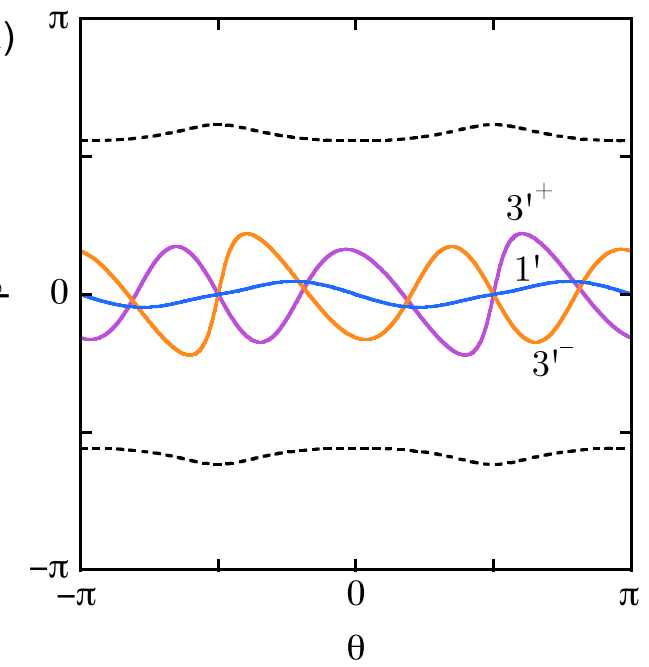

(b)

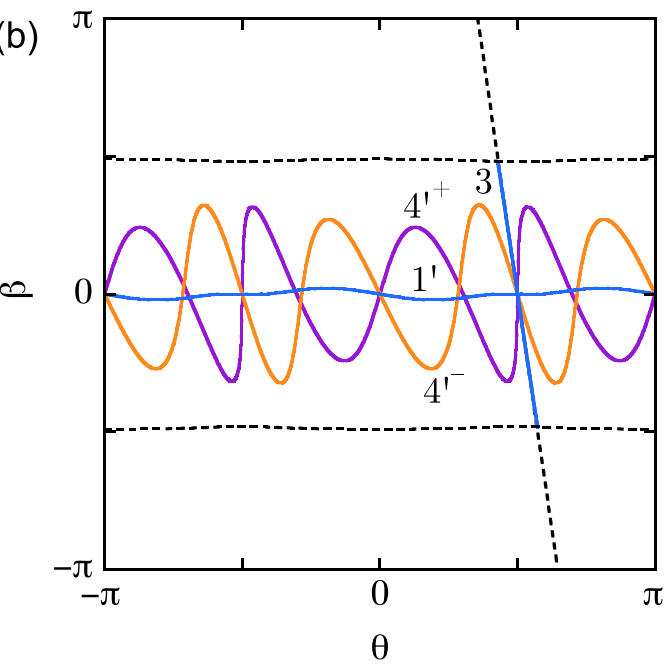

FIG. 14. Plots of $\beta$ vs. $\theta$ for periodic modes with energies $E=0.06$ (a) and -0.01 (b) located initially at $\mathrm{Y}^{ \pm}$(solid traces). In frame (b), the nearly vertical dashed trace gives the relationship of Eq. (109) for small-amplitude oscillations, and the solid trace that overlays it gives the corresponding shape of mode 3. The other dashed traces in frames (a)-(c) give the domain boundaries of Eq. (50).

for Fig. 11, while mode $3^{\prime \pm}$ of Fig. 11 has an antinode at $\theta=\pi / 2$, and is therefore incompatible with the initial condition $\phi(0)=\pi$ for Fig. 14. Modes $1^{\prime}$ and $4^{\prime \pm}$ have nodes at both $\theta=0$ and $\theta=\pi / 2$, so either set of initial conditions reproduces these modes.

\section{CONCLUSIONS}

We have studied the motion of a uniformly magnetized sphere that slides without friction along the surface of a second, identical sphere that is held fixed in space, subject to the magnetic force and torque of the fixed sphere and the normal force. We find two stable equilibrium positions for the free sphere, each with parallel and aligned magnetic moments, and two unstable equilibrium positions, each with antiparallel moments. We use a linear stability analysis to determine the frequencies, amplitudes, and eigenvectors of small-amplitude oscillatory modes near the stable equilibrium positions, and of an unstable oscillatory mode near the unstable equilibrium positions, and determine the domains surrounding these positions to which the free sphere is confined for small energies. We show that these modes remain periodic at finite amplitudes, with frequencies, amplitudes, and eigenvectors that reduce to the linear stability results in the limit of small amplitude. We find that one of the stable modes bifurcates into mixed modes and circumnavigates the free sphere at large energies, and identify an infinite cascade of these bifurcations that cumulates at the same energy $E=-1 / 6$ that the domain becomes contiguous.

Periodic sliding modes offer windows into the rich nonlinear dynamical behavior of this system. Using MagPhyx software, we have identified aperiodic orbits that mimic periodic orbits in some cases and depart from them in others (demos $3,4,5,7$, and 8). ${ }^{20,25}$ We intend to investigate the possibility of chaos in such orbits.

We are also interested in investigating solutions for which the spheres do not slide against each other, but instead experience hard-sphere elastic collisions. Allowing such radial motion (in addition to the orbital and spin motions included in this paper) increases the phase-space dimensionality from 4 to 6, for which MagPhyx reveals a rich array of nonlinear couplings between radial, orbital, and spin motions.

\section{ACKNOWLEDGMENTS}

We gratefully acknowledge support from NSF Grant No. 1332265 and discussions with Charles Jaffe and David Farrelly on the Hamiltonian approach.

${ }^{1}$ See http://zenmagnets.com/gallery/ for The Zen Gallery, curated by S. Qu (last accessed March 8, 2017).

${ }^{2}$ B. F. Edwards, see https://drive.google.com/file/d/0Bw7DdocNZGQgW ThTb3VvUHYza2s/view for Educational value of neodymium magnet spheres: Redacted expert report in the matter of Zen magnets, LLC, CPSC Docket No. 12-2 (last accessed March 8, 2017). See Appendix D for YouTube view counts as of July 18, 2014.

${ }^{3}$ D. A. Richter, see http://www.cpsc.gov/en/Recalls/Recall-Lawsuits/ Adjudicative-Proceedings/ for Expert report "Teaching geometry with magnet sphere kits," in the matter of Zen Magnets, LLC, CPSC Docket No. 12-2, Item 124, Exhibit 3, 10/20/2014 (last accessed March 8, 2017).

${ }^{4}$ J. J. Weis and D. Levesque, "Chain formation in low density dipolar hard spheres: A Monte Carlo study," Phys. Rev. Lett. 71, 2729-2732 (1993).

${ }^{5}$ A. S. Clarke and G. N. Patey, "Ground state configurations of model molecular clusters,” J. Chem. Phys. 100, 2213-2219 (1994).

${ }^{6}$ R. Messina, L. A. Khalil, and I. Stanković, "Self-assembly of magnetic balls: From chains to tubes," Phys. Rev. E 89, 011202 (2014).

${ }^{7}$ C. L. Hall, D. Vella, and A. Goriely, "The mechanics of a chain or ring of spherical magnets," SIAM J. Appl. Math. 73, 2029-2054 (2013).

${ }^{8}$ D. Vella, E. du Pontavice, C. L. Hall, and A. Goriely, "The magneto-elastica: From self-buckling to self-assembly,” Proc. R. Soc. A 470, 20130609 (2014).

${ }^{9}$ N. Vandewalle and S. Dorbolo, "Magnetic ghosts and monopoles," N. J. Phys. 16, 013050 (2014).

${ }^{10}$ J. Boisson, C. Rouby, J. Lee, and O. Doaré, "Dynamics of a chain of permanent magnets," Eur. Phys. Lett. 109, 34002 (2015).

${ }^{11}$ J. Schönke and E. Fried, "Stability of vertical magnetic chains," Proc. R. Soc. A 473, 20160703 (2017).

${ }^{12}$ T. Shinbrot, C. Grebogi, J. Wisdom, and J. A. Yorke, "Chaos in a double pendulum,” Am. J. Phys. 60, 491-499 (1992).

${ }^{13}$ R. B. Levien and S. M. Tan, "Double Pendulum: An experiment in chaos," Am. J. Phys. 61, 1038-1044 (1993). 
${ }^{14}$ E. W. Weisstein, see http://scienceworld.wolfram.com/physics/Double Pendulum.html for Double pendulum, ScienceWorld, 2005.

${ }^{15}$ R. K. Wangsness, Electromagnetic Fields, 2nd ed. (Wiley, New York, NY, 1986), p. 326.

${ }^{16}$ J. D. Jackson, Classical Electrodynamics, 2nd ed. (Wiley, New York, NY, 1975), p. 195.

${ }^{17}$ D. Griffiths, Introduction to Electrodynamics, 3rd ed. (Pearson Education, Delhi, India, 1999), p. 246.

${ }^{18}$ D. J. Griffiths, "Dipoles at rest,” Am. J. Phys. 60, 979-987 (1992).

${ }^{19}$ B. F. Edwards, D. Mark Riffe, J.-Y. Ji, and W. A. Booth, "Interactions between uniformly magnetized spheres," Am. J. Phys. 85, 130-134 (2017).

${ }^{20} \mathrm{~B}$. F. Edwards and J. M. Edwards, "Dynamical interactions between two uniformly magnetized spheres,” Eur. J. Phys. 38, 015205 (2017).
${ }^{21}$ For a similar argument applied to the torque on an electric dipole, see R. K. Wangsness, Electromagnetic Fields, 2nd ed. (Wiley, New York, NY, 1986), p. 127.

${ }^{22}$ S. H. Strogatz, Nonlinear Dynamics and Chaos (Addison-Wesley, New York, 1994), p. 128.

${ }^{23}$ W. H. Press, S. A. Teukolsky, W. T. Vetterling, and B. P. Flannery, Numerical Recipes in Fortran: The Art of Scientific Computing, 2nd ed. (Cambridge University Press, New York, NY, 1992), p. 704-708.

${ }^{24}$ W. H. Press, S. A. Teukolsky, W. T. Vetterling, and B. P. Flannery, Numerical Recipes in Fortran: The Art of Scientific Computing, 2nd ed. (Cambridge University Press, New York, NY, 1992), p. 352-355.

${ }^{25}$ J. M. Edwards, see http://www2.cose.isu.edu/ edwajohn/MagPhyx for MagPhyx Software (last accessed March 11, 2016). 